786 Wermann, Leber die dureh Berührung der Primula obconica

Ich habe die Pillen in 4 Fiallen angewendet, und der Erfolg entsprach dem der ïblichen Sublimatpillen (0,3 Sublimat auf 60 Pillen), ohme dass starke Nebenwirkungen aut den Darm, wie sie bei jenen üblich sind, hervorgerufen wurden. Stomatitis trat in keinem Falle auf.

Als Ergebnisse meiner Beobachtungen möchte ich folgende Ceberougungen aussprechen:

1. Das Hyrgol besityt keine besonderen Vornüre, die in therapentischer Hinsicht eine Bevor\%ugung vor dem alten unlöslichen Q Quecksilber rechtfertigen kïmuten.

2. Fïr die subkutane Einverleibung ist die wissrige Lösung des Hyrgol nur mit Vorsicht an\%uwenden, erstens mit Riicksicht auf die noch nicht feststehende Valen\% des Mittels, \%woitens wegen der S'Chmerzen bei und nach dem Injicieren, drittens der Neigung halber schwer resorbierbare Infiltrate \% bilden.

Nïhere Untersuchung physiologisch-anatomischer Art sind noch erforderlich, um nach\%uweisen, ob das ohne lïweiss\%usat\% dem lï̈rper einverleibte Hyrgol im Gewebe in das unlïsliche Merkur rerwandelt wird, beziehentlich chemischer Art, um fest\%ustellen, ob die Kïrpersiitte diese Lmset\%ung \%u Stande \%u bringen vermiggen.

3. Bei der Schmicrkur sind niederprozentige Salben als die offimelle anzuwenden. Die Wertigkeit des Hyrgols scheint höher \%u sein, als die des alten (quecksibers.

4. Die Hyrgolpillenkur ist nach bisherigen Erfahrungen die greeignetste Art der 1)arreichung.

\title{
Jiteratur:
}

1. A. Jottermoser: Ueber colluidales Quecksilber. (Jomrnal für prakt. Chemie 1S!s: Bd. in.

2. Werler: Ueher Anwendungsweise und Wirkungendes bïslichen metallisehen Quecksilbers. (Berlin. klin. Nochensehrilt, 18!s, No. 1‥)

3. Itochnel: Entersuchungen des colloidalen Hydrargrum des

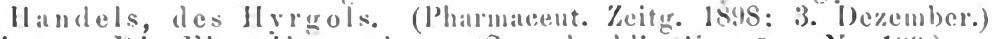

4. Neisser: Die Einreilungskur. (Sammlge. klin. Vortrige, No. 1!!9.)

b. Werther: Hrdrarurum eolloidale als Antisphiliticum. (Monatsh. f. !rakt. Dermattol. Is!s; 1.). De\%.)

\section{XXVII. \\ Ueber die durch Berührung der Primula obconica entstehende Hautentzündung.}

Von

\author{
I) med. ERSST WELMAN
}

in I)resden.

Die erste" Mittheilung äber "Dermatitis durch Berïhrung einer Primela erfolgte von Riehl') im .Jahe 1S95, welcher die-

1) Wien. Klin. Winschr. VIII. 11. 18!);. 
selbe bei cinem Gïrtner beobachtete. The hantreionde Wirkung der Primula obconica, um diese handelto es sich, war den Gïrtnern bekannt und hatte die Entfernung der Pflan\%e aus den Sichönbrumner Glashäusern voranlasst, da die durch sie verursachten Spitalkosten \%u hoch geworden waren.

Ich hatte Gelegenleit, die Erkrankung in einigen Fällen in der Privatpraxis bei Patientimnen \%u sehen, welche die genamnte Vierpflan\%e im Vimmer gepllegt hatten; sie erkrankten mit einer heftigen Hautent\%ïndung der Hände und des Gesidhts, olmo dass die Irsalde des Taidens festerestellt wurle und set\%ten sirlo in Folge dessen, olme es $\%$ wissen, immer wieder dor Schäidlichkeit aus, so dass sie wiederholte Infälle des quälenden leedens \%u bestehen hatten. Dal die Nitur einer derartigen primeldermatitis" demnach leicht äbersehen wird, möchte ich kur\% auf das charakteristische Krankheitsbild eingehen und als Beispiel einen sehr pröinanten Fall mittheilen, äber welchen ich im oktober 1896 in der Gesellschaft für Natur- und Heilkunde in Uresilen berichtet hible:

Ende $\Lambda$ ugust 1896 wurde mir durch die Güte der Herren Med.-Rath J)r. Niedner und I)r. Matennel eine jejälurigr I)ame \%ugewiesen, welche seit längerel \%oit von einer eigenthïmlichen Hauterkiankung befillen war. Sie hatte trüher ilire gam\% gresunde Haut grehabt und inshesondere bisher nie an einer äln-

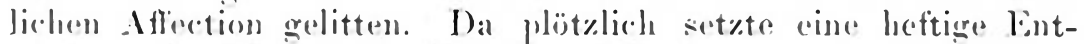
zündumg der Haut beider Hände und Vorderarme oin mit lï̈thung

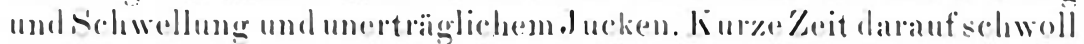
anch das (iesicht an, so dass die l'atientin gan\% rutstellt wurde; dic Gesichtshaut wall dunkeleoth und ansserordentlich empfindlich, die Dugen begamen \% thränen und die Kranke hatte 'lage und Dilcht keine liuhe. Allmählied besserte sich jedoch wieder

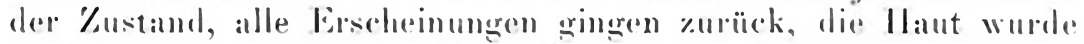

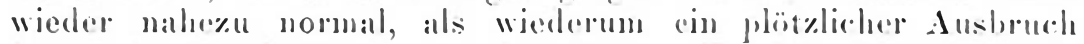

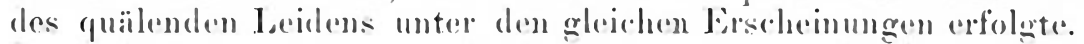
So wiederholte sich unter bald wieder eintretender Besserumg. bald wioder neuer Verschlimmerung das kiranklacitsbild, und die l'atientin kam schliesslich in einen /oustand hocheradierer Nore rositat und Uiruhe.

As ich die Dame sah, hatte sio soeben wieder einen neuen Anfall gohabt. Die llaut heider Handräcken und Vorderarme wall dunkel greröthet, polsterartig geschwollen und befind sich in einem ansserordentlichen Rei\%ustande; im Gesicht wal die Lmgobung des Mundes, der Sase und der Augen ebentalls geriithet. und stark ödematois gescolwollen. Die Patientin laatte in Folece

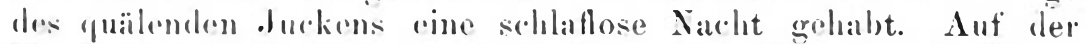

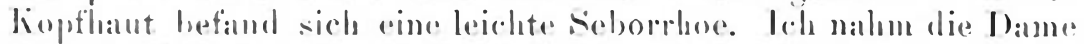
in meine lilinik anf und liess sofort eine lionpinsolung mit Kink-

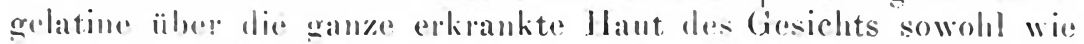

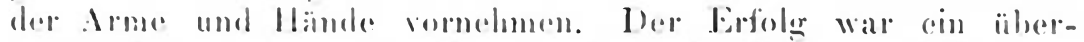

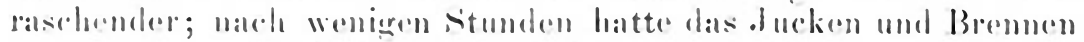

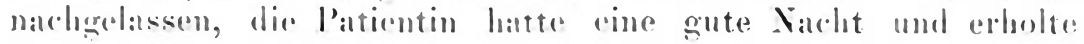


788 Wermann, Ueber die durch Berïhrung der Primula obconica

sich in den nächsten 'Tagren vällig̣. Mls ich nach 3 'Jagren den Kinkleimverband entfernte, war ich erstaunt, eine nahe\%u normale Haut vor mir \%u haben, die Rïthung und Sichwellung war fast rollstaindig rerschwunden und hatte einer leichten $A$ hschuppung Plat\% gemalcht. Einige 'lage spaiter entliess ich die Patientin, la in\%wischen vïllige Restitutio ad integrum eingetreten und kein nener Anfall erfolgrt war. Joh konnte es mit um so besserem Gewissen thun, als ich in\%wischen die Lrsache des Leidens festgestellt hatte und die Prognose in Folge dessen eine günstige greworden war. Ich täuschite mich darin aluch nicht, denn die Patientin war und blich gesund und hat auch bis \%um heutigen 'Tage, wie sie mir auf Befragen mittheilte, nkeinerlei Halutbelästigung wieder gehabt".

Dic gan\% Art des Verlatufes der Frkrankung, die rasche und durch keine /wischenfälle unterbrochene Wiederherstellung in der libinik legte ron rornherein den fredanken nalue, dass das Iseiden der Patientin der Finwirkung einer in ihrer Behausung sich rorfindenden soluädlichkeit \%n\%schreiben sei. Die nähere Nachforschung ergah) denn aluch, dass die Dame in ilurem Kimmer. einen chinesischen Primelstock hatte, welchen sie, da er ilnt durch präclitiges fiedeihen viel l'rende machte, selar sorgsalm gepllegrt hatte; nach lintfernung der Primula obconical, denn um eine solche handelte es sich, war die P'atientin dauernd ron ihren Iscilen betreit.

Meine Aufmerksamkeit war zuerst anf die Primeldermatitis durch einen Fall gelenkt worden, der die frau eines ausürtigen Giartnereibesit\%ers betrat; dieselbe hatte wielerololt an heftigen Hautent\%ündungen der Hände und hesonders der Finger \%u leiden. Wie mir ihr Mann später mitheilte, war die Primula obconica die Irsalche gewesen; so oft seine Frau mit dieser Phan\%e, welche er im Crossen \%üchtete, in Borïhrung grokommen wall, war sie erkrankt. Sie trug nummehr Handschuhe bei dieser Crelegenheit und die Hatutent\%ündung War ron stund an verschwunden.

Charakteristisch für die Primeldermatitis ist demnach plït\%liches, anfallartiges Aufteten einer leftigen Hatent\%ündung \%uerst an den lländen, alsiann im Cresicht bei Personen, die sich berullich oder alus Liebhaberei mit Blumen\%ucht beschäftigen und dabei mit der Primula obconica in Beriilarung kommen; ferner starke Röthung und sichwellung der ererriftenen Parthien, in manchen Fällen sograr Blasenbildung (Richl), beträchtliche Betheiligung des Allgemoinbefinclens, rerhälnissmässigr rasche Besserung bei antiphlogistischer behandlung, leichtes Auftreten von Recidiven, sobald die P'atienten \%u ihrer gewohnten Lebensweise zurïckkchiren.

Die hautrei\%ende Wirkung wird durch eine farblose Flïssigkeit hervologebracht, welche in kur\%n an der Oberlläche der Bläitter sit\%enden Drüsenhaiaren enthilten ist und bei Borührung der l'flan\%e anstritt. Die Wirkung tritt nicht sofort aut, sondern kommt erst nach längerer \%eit \%um Vorschein, so dass die l'at- 
tienten nirlit von sellust auf die Vermuthung kommen, dass ihr Leiden ron der Berülarung der Primel herkomme.

Wie Herr Prof. Dr. Irude, Director des Dresdener botanischen Gartens mir mitmutheilen rie Giite hatte, ist von dieser hautreimenden Eigrenschaft der Primula obconica in gärtnerischen \%eitschriften wielerholt die Rede gewesen, lie Gärtner bestritten aber dieselbe, weil die meisten Menschen gan\% unempfindlich dagegen soien; er selbst habe, wie auch sein Assistent, längere /eit bei der Anstellung ron Kä̈litungsversuchen wiederloolt die Pllan\%e in Händen geolabt, olme dass sich die geringste binwirkung anf dic Haut bemerkbar gemacht labe; dagegen liabe die Primel bei seinem \%weijährigen Sïhnchen einen leichten rasch vorïbergegangenen Ansschlag an den Händen hervorgerufen. Ich selbst, wio anch das Personal meiner Klinik und eineelne Patienten, deren Haut der Einwirkung der Primel ausgeset\%t wurde, \%eigten sich unempfindlich dagegen, nur in einem Falle war eine ganz geringe nur kure Keit andauernde Hautrei\%ung entstanden; sellist bei dem 20jährigen sohne der Eingargs erwähnten Patientin, welcher seit Kindheit an häufig recidivirender Irticaria litt, rief die absichtlich vorgenommene Einreibung der Haut mit den Blättern der Primel keinerlei Hautent»ündung hervor.

lis gehört demnach eine hesondere Disposition der Haut da\%u, dass die Primula oboonica ilure reizende Wirkung entfaltet. Geijl und ran Praa $\left.\underline{G}^{1}\right)$ beschricben einen fiall, in welchem eine Genitalerklankung (chronische Metritis mit Cervixriss und alter Dammriss), die Haut in einen Kustand gebracht hatte, welcher dem Gift Gelegenheit bot, seine Wirkung \%u entfalten. Die Patientin hatte eine selur reizbare Haut und bot die Keichen der Dermographie dar. Arctander.2) beohachtete die Primeldermatitis bei einer Dame, bei der ein Jahr vorher die Berührung einer Nessel eine heftige Hautent\%ündung des Armes hervorgerufen hatte. Er hat die lirkrankung nur bei Frauen gesehen und salubt diher, dass das weibliche (reschlecht eine besondere D)isposition dafïr besitze; eine (iärtnersfran latte starkes Jucken, besonders des Naclits, wenn sie am Tage mit der Pflan\%e \%u thun grehabt hatte; eine ailtere Schwester der Patientin, welche an Expom des Gesichts und anderer Körperstellen geliten hatte, bekam \%wei Stunden, naclidem sie einige Primelblatter alogepflückt hatte, Frösteln und Jucken an den Händen, starke Rüthung des Gesichts und Schohwellung der Lider.

In den rällen, die ich beobachtete, konnte ausser einer Sebormoe der Kopthaut keinerlei Inomalie nachgewiesen werden; vielleicht genügte der seborrhoische Kustand da\%u die Haut für das Primedgitt empfäuglich \%u machen.

In der Regel verschwindet nur nach Entfernung der Primel die Hatutent\%ündung dauernd. Fine Beobachtung möchte ich

1) Monatshefte für prakt. Dermatol. XXII. p. 173, 1896.

2) Anmales de Dermatol. et de Syph. VIII. p. 1100, 1897.

Dermaiologlscbe Zcitschrift. Bd. V. Heft 6. 
jedoch nicht verschweigen, welche in ein\%elnen Fällen auch einen anderen Ausgang des lueidens wahrscheinlich macht. lis sind mir hartnäckige Falle von seborrhoischem Ec\%em vorgekommen, welche mit heftigem .Jucken begonnen hatten, und in denen der Zusammenhang mit einer Primeldermatitis sehr wahrscheinlich war, wenn er sich auch mit Sicherheit nicht mehr feststellen liess, da der Beginn des Lueidens sehr weit \%urücklag, und die Patientimnen ilue Primel, die sie seiner \%eit gepflert, nach dem Abblühen entfernt latten. Der Gedanke lässt sich nicht von der Hand weisen, dass bei da\%u disponierten Personen eine derartige Primeldermatitis den Ausuangspunkt für ein hartnäckiges und der 'Therapie oft lange Keit Widerstand leistendes scborrhoisches Jo\%em bilden kamm, welches namentlich durch heftiges Juckeu sich bemerklich macht.

Mit Erlaubniss des Autors füge ich obiger Mitteilung die Bemerkung hinz, dass ich ïber eine gleichlautende Beobaching verfüge. Eine lame rom lounde, grosse Blumenfreundin, litt wiederholt an anscheinend san\% räsellatier Dermatitis urticans, bis in dem Einfluss der Primula obconic:al die Ersalche des leeidens erkannt und dauernd beseitigt werden konnte.

ber Heransugeber.

\title{
XXVIII.
}

(Aus Professor Lassars Klinik.)

\section{Zur Histologie der Klauenseuche. (Dermatosis zoonotica.)}

von

\author{
Dr. TH. II I Y ER,
}

Assistenten der kininik

(llier\%"litiel X

\begin{abstract}
Von der bei Tieren so hitufigen und nach allen Untersuchern sicher infektiösen lirankheit sind Übertrarungen auf den Menschen nur in relativ spärlicher Kahl bekannt geworden. Der erste, welcher die Bechlochtung machte, dass Menschen nach Genuss ron Milch senchenkranker lïihe an Sohlingbeschwerden, gesteigrepter Hit\% in Mund und Rachen und Aphthenbildung erkiankten, ist Sagar (17(it). 1500 folgte ilum Brosche mit ähnlichen Mitteilungen, während 18331 Hertwig als erster experimentell an sich selbst die C̈bertragbarkeit der Contagrums auf den Menschen darthat. Yon neucren

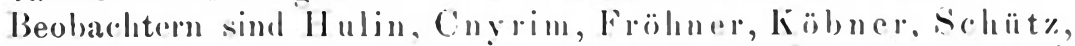
Ellenbererer \%u nemnen und namentlich hat śiegel anlissslich cince in der Umerebung ron Brit\% herrsehenden Eprootie ron

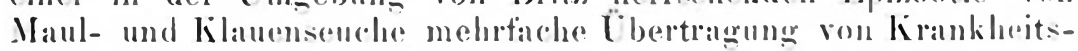
erscheinungen aut den Menschen geselaen und grenan boschrieben. Ausserdem haben in letoter \%eit Sirheyer, libstein (I). M. Wschrt. 1s!)(;, No.!) u. 10), sichlatter (Boitr. \% klin. Chirurgie,
\end{abstract}

